\title{
NON-NEWTONIAN EFFECTS IN BLOOD FLOW SIMULATIONS OF CORONARY ARTERIAL FLOW
}

\author{
Alex J. Apostolidis, Adam P. Moyer ${ }^{1}$ and Antony N. Beris ${ }^{2}$ \\ Department of Chemical and Biomolecular Engineering, \\ University of Delaware, Newark, DE 19716
}

\begin{abstract}
SYNOPSIS
We present a careful evaluation of non-Newtonian blood rheology effects in arterial flow simulations. We achieve that by comparing the converged solutions obtained a) from a Casson viscoplastic modeling of blood rheology using a recently developed parametrization [Apostolidis and Beris, J. Rheol., 58: 607-633 (2014)], and b) from a Newtonian model. We emphasize on the proper implementation of outlet boundary conditions (OBCs) in a way that ensures consistency with the pressure/flow predictions of the downstream network, which is modeled approximately using a 1D model [Johnson et al., Comp. Chem. Eng., 35: 1304-1316 (2011)]. We further improve and validate the iterative scheme proposed by [Johnson et al., Int. J. Num. Meth. Fluids, 66: 1383-1408 (2011)] for the implementation of the OBCs, by employing it in conjunction with a) Casson-derived simulation data, b) a more accurate geometrical model and c) an accelerated convergence iterative scheme through application of Shanks transformation. Finally, we performed a rigorous analysis to ensure appropriately converged solutions.

Our investigation shows significant differences (up to 50\%) between the simulation output of Newtonian and non-Newtonian models. The differences are attributed to the coupling that exists between low and high shear rate areas in the flow. They show the significance of nonNewtonian blood rheology and motivate further work towards an even more accurate modeling of the thixoropic and three-dimensional characteristics of the blood flow rheology that go beyond the Casson model utilized in the present work.
\end{abstract}

KEYWORDS: Blood Flow, Simulations, Coronary Arterial Flow, Casson Model, Outlet Boundary Conditions.

1 Current address: Molecular Engineering and Sciences Institute, University of Washington, Seattle WA 98195

2 Author to whom correspondence should be addressed 


\section{Introduction}

In the past two decades CFD has given rise to a large number of medical-related studies, such as the analysis of the flow in specific arteries. Cardiovascular diseases are affected by the hemodynamics within the arteries, as low wall shear stress values (WSS) have been correlated with atherogenesis, the initial stages of atherosclerosis [Cecchi et al. (2011)]. Therefore, CFD can be used to obtain the WSS distribution in the vessels of investigation.

Despite the rapidly increasing number of such investigations, it can be argued that CFD biomedical applications are still limited [Byoung-Kwon (2011)] rendering the physiological relevance of hematological data acquired from simulations questionable. The commonly accepted limiting factors that decide the accuracy of the results of CFD simulations in the biomedical field are the accuracy of the geometric model, the complexity of fluids in the human body, and the imposed boundary conditions (BCs) [Byoung-Kwon (2011)].

Medical imaging techniques, such as computed tomography, ultrasound imaging, and magnetic resonance imaging offer very detailed, personalized geometrical models that can be used for CFD simulations. Therefore, the geometric representation no longer constitutes a significant limitation. Similarly, Doppler ultrasound, pressure wire, and other non-invasive techniques, provide pressure and flow information at specific locations of the arterial network, which then can be used as BCs in CFD simulations. However, the information obtained in each case reflects specific data of a specific case, at a specific time. These technologies therefore cannot be used to examine what-if scenarios for modeling practices [Johnson et al. (2011a); Taylor and Draney (2004)]. Thus, imposing the appropriate BCs in simulations of arterial flow requires special attention.

The proper implementation of outlet BCs in simulations of flow in a specific artery requires that the impact of the rest of the arterial network be taken into consideration [Formaggia et al. (2009)]. This can be achieved by using boundary conditions that, instead of absolute pressure or velocity values, describe a correlation between outlet flow and pressure [Johnson et al. (2011a); Formaggia et al. (2009)]. Such correlations represent flow information for the arterial network that extends beyond the boundary points of the simulation. The two main approaches that have been used for the implementation of more sophisticated boundary conditions are only distinguishable based on the complexity of the network model involved and they range from a linear resistance or lumped parameter/Windkessel (0D) model to a 1D full arterial network [Johnson et al. (2011a)]. The second approach, which bases the outlet BCs on the predictions of an approximate flow model for the entire arterial network, is more rigorous and became more popular in the past decade. However, it requires a sophisticated coupling of the $1 \mathrm{D}$ model predictions to the $3 \mathrm{D}$ simulations. One way this coupling can be made possible is to directly incorporate them to the numerical simulations, through the use of proprietary, inhouse developed, codes. Detailed discussions on the coupling between the $3 \mathrm{D}$ domain and the 
lower order models can be found in literature [Johnson et al. (2011a); Formaggia et al. (2009); Esmaily-Moghadam et al. (2013); Formaggia et al. (2001)].

Recently, Johnson et al. (2011a) proposed a more versatile approach that allows the implementation of the network outlet boundary conditions into commercial CFD codes, such as FLUENT. It achieves that by decoupling the detailed 3D simulation upstream from the more approximate 1D network downstream. The 1D model-developed flow-pressure correlations are represented in the form of complex impedance coefficients in the Fourier domain [Johnson et al. (2011b)]. Those relations are then taken into account separately, decoupled from the 3D simulation, within an approximate "simulant" model (run in MATLAB environment). However, as the correction term used in the simulant is flow-dependent, iterations are needed until convergence, based on the output flowrate results from the $3 \mathrm{D}$ simulation. This scheme was first demonstrated with simulations of the flow in the left coronary artery [Johnson et al. (2011a)]. However, the geometric model used in that study was simplistic and not capturing important effects that can impact the dynamics of the flow, such as the curvature of the vessels. Moreover, the simulations were not shown to fully converge and there were no systematic convergence studies of the numerical parameters involved. In addition, the calculations were based solely on the Newtonian assumption of the fluid, therefore neglecting the complex rheology of blood [Apostolidis and Beris (2014); Apostolidis et al. (2015)]. The present study has as objective to rectify all these limitations.

The complex nature of the fluids in the human body is a main reason of concern for the reliability of the results of blood flow simulations. For the simulations of the human right coronary arteries, Johnston et al. (2006) showed that, "when studying the wall shear stress distribution for transient blood flow in arteries, the use of a Newtonian blood flow model is a reasonably good approximation. However, to study the flow within the artery in greater detail, a non-Newtonian model is more appropriate". The non-Newtonian characteristics are prevalently demonstrated at low shear rates which can exist near bifurcation sites and at recirculation zones developing in the arteries. Since the generation of atherosclerosis, i.e. atherogenesis, has been correlated with low ( $<0.5 \mathrm{~Pa})$ WSS values [Cecchi et al. (2011); Chaichana et al. (2012); Soulis et al. (2008); Wentzel et al. (2012)], it becomes clear that non-Newtonian models need to be employed in order to obtain more reliable estimates.

Non-Newtonian models have been employed with increasing frequency for flow simulations of various vascular components, such as the aorta [Karimi et al. (2014); Morbiducci et al. (2013); Liu et al. (2011)], the cerebral [Bernabeu et al. (2013); Campo-Deaño et al. (2015)], and the coronary arteries [Johnston et al. (2006); Lassaline et al. (2014); Chaichana et al. (2012); Soulis et al. (2008)]. In all of these cases, generalized Newtonian expressions are adopted which can better capture the complex behavior of the fluid under steady state conditions. However, while there is strong evidence to support that the Casson model is the most suitable among constitutive equations for the description of blood flow faithfully, reproducing both its yield 
stress and shear thinning viscoplastic characteristics [Apostolidis and Beris (2014)], researchers often employ different models such as the Carreau-Yasuda [Lassaline et al. (2014); Bernabeu et al. (2013)] or a general power law [Chaichana et al. (2012); Johnston et al. (2006)]. The differences in the predictions of various rheological models are presented in a comparative study of Karimi et al. (2014).

From all of the above there is accumulating evidence collected on the importance of a non-Newtonian rheology to arterial flow. However, the degree of the effects of the rheology is still debatable as the data comparing the non-Newtonian to Newtonian effects have been often generated a) in highly approximate geometries and/or b) under steady-state or time-dependent conditions but with fixed outlet boundary conditions and/or c) with inappropriate model and/or d) without demonstrating convergence with mesh and other important numerical parameters. Thus, the need arises to a study that removes these degrees of uncertainty. Regarding the nonNewtonian blood flow characteristics the dominant ones are the shear thinning and viscoplastic properties that can be most easily described by a generalized non-Newtonian model. Although from long time researchers have recognized the time-dependent thixotropic characteristics of blood rheology [Bureau et al. (1980)] the best so far available models are still restricted to shear flows and come with parameters with values that are not as yet fully connected to blood physiology [Apostolidis et al. (2015)] leaving considerable degrees of uncertainty in their proper determination. However, as thixotropy tends to decrease the non-Newtonian purely viscous effects, such as described by a generalized Newtonian model, the use of the latter as an approximation for any type of flows is still valuable and can be considered as placing an upper bound on the magnitude of the evaluated non-Newtonian effects.

We believe that for the proper evaluation of the non-Newtonian rheology effects, in addition to a proper non-Newtonian description, special care needs to be placed to assure that a) both Newtonian and non-Newtonian simulations have produced converged results (both with respect to the mesh but also with respect to any other numerical parameters, such as the time-step size) b) a reasonably faithful arterial flow geometry is used and c) proper outlet boundary conditions are specified. This is exactly the goal of the present work, where we have selected the first bifurcation of the left main coronary artery as the model geometry, the Casson model and the parameterization developed recently [Apostolidis and Beris (2014)] to represent healthy blood as the non-Newtonian model to test again the Newtonian fluid predictions, and the Johnson et al. (2011a) iterative, decoupled, method of implementation of the outlet BCs.

A secondary goal of the present investigation is to further improve, through a Shanks convergence acceleration method, the convergence of the iterative method for the outlet boundary conditions (OBCs), as well as to more extensively validate it, using either Newtonian or non-Newtonian rheology and applied to a more faithful representation of the arterial geometry. The OBCs are obtained from a 1D-network model of the arterial system [Johnson et al. (2011b)], while the system of investigation is the left coronary artery (LCA) bifurcating into 
the left anterior descending (LAD) and the left circumflex (LCX). The geometric model used in the present work includes significant improvements to the previously developed geometries that were used in the past when the simulant-based approach for the efficient implementation of OBCs was first introduced by Johnson et al. (2011a).

The rest of the paper is organized as follows: in Section 2 we discuss the materials and methods used; this entails the construction of the geometrical model, an overview of the Casson parametric model for blood flow, a briefing of the simulant model, its numerical implementation through an iterative and the acceleration of the scheme's convergence via numerical analysis. The results of the convergence studies and the comparison between the Newtonian and Casson simulations results are presented in Section 3. Finally, Section 4 has our conclusions.

\section{Flow Geometry, Rheological Model and Numerical Methods}

\subsection{Flow geometry}

The present investigation regards the examination of a prototype 3D arterial flow problem. As the particular simulated vascular geometry used in this study we chose the left coronary artery (LCA), which consists of the left main artery (LM) that bifurcates into the left anterior descending (LAD) and the left circumflex (LCX). The constructed geometrical model, a simplified prototype asymmetric arterial bifurcation, is presented in Figure 1 along with one of the meshes used in the numerical simulations.

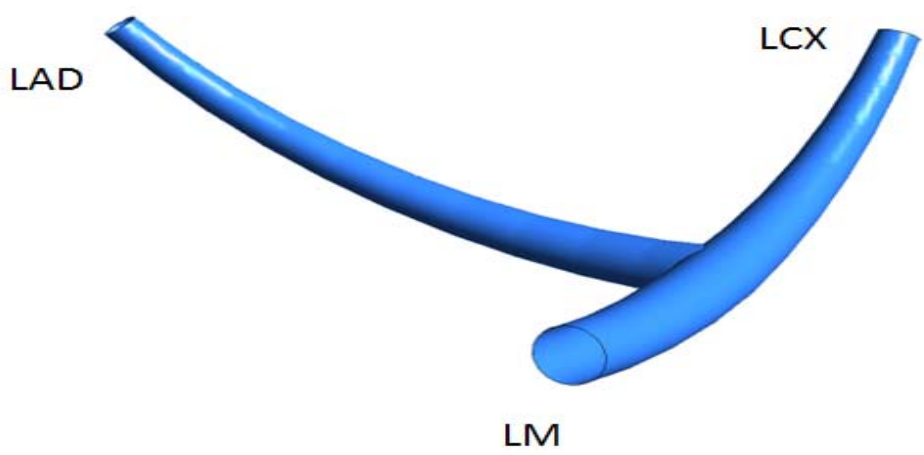

(a) 
(b)

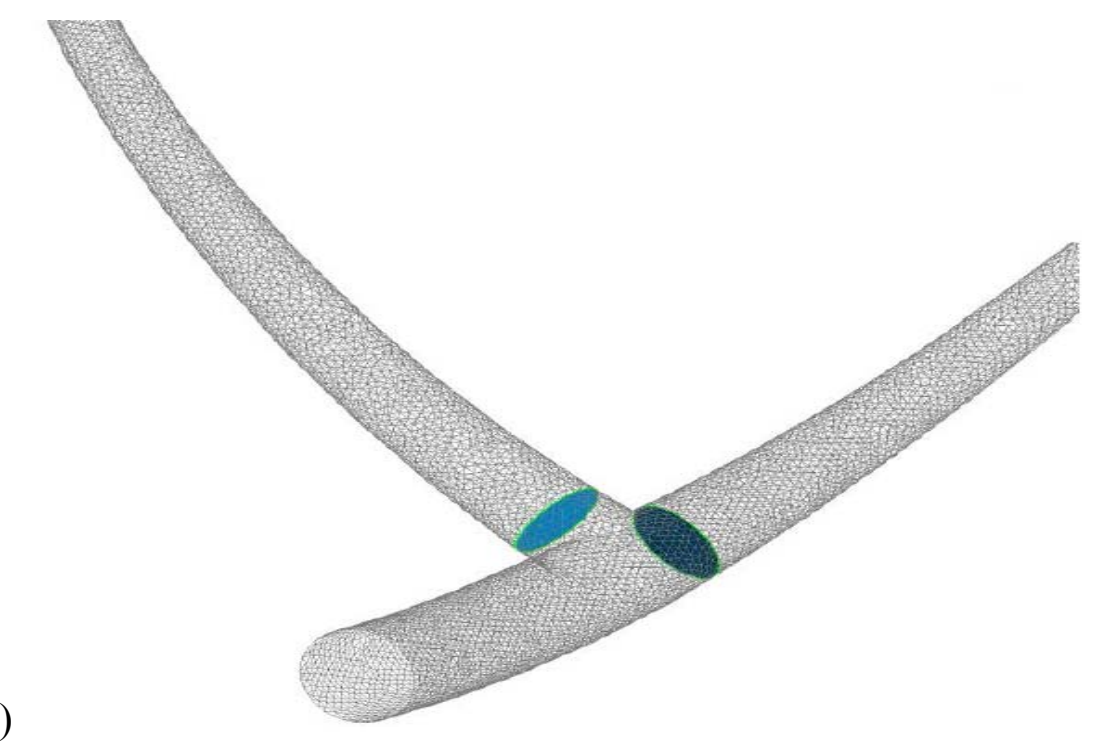

Figure 1. (a). Simplified constructed geometry of the left coronary artery used for the simulations, (b). One of the meshes (base density) that were created for the simulation; two cross-sections that were used for data analysis, near the bifurcation site, are also highlighted

The simplified vascular geometry has been developed based on anatomically accurate dimensions of the LCA, as reported in literature [Dodge et al. (1992)]. The dimensions of the constructed vessel geometry are presented in Table 1. Tapering effects were included in the construction of the LAD and LCX arteries, while a branching angle of $90^{\circ}$ was used between the LCX and the LAD [Dong et al. (2015)]. Finally, an important feature, and an improvement to the previously used geometries of our LCA investigations [Johnson et al. (2011a)], is the inclusion of curvature in the geometry vessels. The curvature originates from the modeling of the heart as a sphere, therefore requiring that the arteries existing on the surface of the heart be curved as well.

The geometry as well as the meshes were developed with the use of the commercial software ANSYS ICEM CFD version 12 (ANSYS, Inc., Canonsburg, PA, USA). 
Table 1. Characteristic lengths ( $\mathrm{mm})$ of geometrical model

\begin{tabular}{|c|c|c|c|c|}
\hline$\overline{~ V e s s e l ~}$ & Diameter & & Length & Curvature Radius \\
\hline & In & Out & & \\
\hline LM & 4 & 4 & 10 & 34.92 \\
\hline LCX & 3.4 & 1.6 & 17 & 34.92 \\
\hline LAD & 3.8 & 2 & 30 & 34.92 \\
\hline
\end{tabular}

\subsection{Physiologically evaluated rheological parameters}

The minimum physiological parameters of importance in simulations of blood flow are the temperature, $T$, the hematocrit, $H c t$, and the fibrinogen concentration, $C_{f}$ [Apostolidis and Beris (2014)]. Fibrinogen is known to play a key role in bridging the adjacent red blood cells (RBCs) and therefore giving rise to $\mathrm{RBC}$ aggregates. The presence of $\mathrm{RBC}$ networks explains the complex rheology of blood, manifested by the exhibition of yield stress, a property that is strongly dependent on $c_{f}$. The results obtained in the current investigation correspond to average, normal values of physiological parameters

$\left(H c t=40 \%, T=37 \mathrm{C}, C_{f}=0.3 \mathrm{~g} / \mathrm{dl}\right)$.

We have carried out simulations for blood density of $1060 \mathrm{~kg} / \mathrm{m} 3$ both under the Newtonian assumption, and by taking into consideration the non-Newtonian rheology of blood, in the form of a Generalized Newtonian model, the Casson model, capable of representing its yield stress (viscoplastic) characteristics along the shear thinning ones [Apostolidis and Beris (2014)]. In the first case, a typical constant viscosity, $\mu$, of 0.003 was used. In the second, we employed a parametric form of the Casson constitutive equation that we recently developed [Apostolidis and Beris (2014)]. This model has been extensively validated against experimental data, while comparisons with other models frequently used in blood flow simulations, such as the Herschel Bulkley and the Bingham model, show that the Casson describes best the rheology of blood [Apostolidis and Beris (2014)]. The Casson parametric model is defined as: 


$$
\begin{gathered}
\sqrt{\tau}=\sqrt{\tau_{y}}+\sqrt{\mu \dot{\gamma}}, \tau \geq \tau_{y}, \\
\tau_{y}=\left\{\begin{array}{cc}
{\left[\left(H c t-H c t_{c}\right)^{2} \times\left(0.5084 c_{f}+0.4517\right)^{2}\right.} & H c t>H c t_{c} \\
0 & H c t \leq H c t_{c}
\end{array}\right. \\
H c t_{c}=\left\{\begin{array}{cc}
0.3126 c_{f}{ }^{2}-0.468 c_{f}+0.1764 & c_{f}<0.75 \\
0.0012 & c_{f} \geq 0.75
\end{array}\right. \\
\mu=\eta_{p}\left(1+2.0703 \times H c t+3.7222 \times H c t^{2}\right) \times \exp \left(-7.0276\left(1-\frac{T_{0}}{T}\right)\right),
\end{gathered}
$$

where $\tau_{y}, \dot{\gamma}$, and $H_{c t}$ denote the yield stress, shear rate, and critical hematocrit, respectively. The critical hematocrit is the minimum hematocrit below which blood does not exhibit a yield stress. In this model, $\eta_{P}$ represents the plasma viscosity and has a value of $1.67 \times 10^{-2}$ dynexs $/ \mathrm{cm}^{2}$, while $T_{0}$ is the reference temperature of $296.16 \mathrm{~K}$. For the physiological parameters reported above ( $\left.H c t=40 \%, T=37 \mathrm{C}, C_{f}=0.3 \mathrm{~g} / \mathrm{dl}\right)$, the model predicts a yield stress of $0.00412 \mathrm{~Pa}$ and a viscosity of $0.00295 \mathrm{~Pa} \cdot \mathrm{sec}$. Note that the Casson model viscosity value is about the same as the typical Newtonian viscosity that is typically used for blood and we have also used here for comparison purposes, the difference between the two models being restricted therefore in the presence or not of a yield stress.

\subsection{Efficient implementation of outflow boundary conditions (OBCs)}

While the inlet boundary condition is a periodic mass flux (period $\mathrm{T}=1.25 \mathrm{sec}$ ) readily obtained from Johnson et al. (2011a), and the typical no slip condition is applied on the vessel wall, the proper implementation of the outlet pressure BCs requires some attention. The complexity rises due to the inclusion of the proper closed network condition in the simulation of blood flow through the LM coronary artery system, which requires that the outlet pressure profiles are related to the outward flow rates in a fashion consistent with the rest of the network. The pressure/flow information for the vasculature that extends beyond the limits of the 3D simulated geometry are obtained from a 1D-network model [Johnson et al. (2011b)], described in the form of complex impedances:

$$
\hat{P}_{j, k}=\hat{Z}_{j, k} \hat{Q}_{j, k},
$$


where $\hat{P}_{j, k}, \hat{Q}_{j, k}$ and $\hat{Z}_{j, k}$ are the $k$-th Fourier modes of the pressure, flow rate and the complex impedance, respectively and the subscript $j$ denotes the vessel of reference (LAD or LCX). Note that the zero-th order term represented the average (mean) flow resistance with the corresponding pressure describing the mean difference from the average capillary pressure and the complex impedance represented by an equivalent real viscous resistance. The 1D-network model uses analytic solutions for the mean and the time-periodic components, based on lubrication approximation and the Womersley solution of viscous oscillatory viscous flow within elastic walled vessels [Womersley (1955)], respectively, for the blood flow throughout the whole arterial network. The 1D model is constructed based on a detailed geometric information for the principal 45 main arteries [Olufsen (2004)] and scaling relations for the rest of the vessels [West (1999)] going all the way to the capillaries incorporating a total of more than 20 million vessels [Johnson et al. (2011b)].

The consistent implementation of the 1D network model-originated outlet boundary conditions within a commercially available CFD software (here: FLUENT) is achieved through the use of an approximate simulant model of the outlet pressure/flow relationship corresponding to the full 3D and time-dependent numerical FLUENT simulations, based on the original development of Johnson et al. [2011a], where one can also find a detailed description of the proposed scheme. A brief explanation of the simulant model and its implementation scheme are discussed below in Sections 2.3.1 and 2.3.2, respectively.

\subsubsection{The simulant model}

Johnson et al. [2011a] proposed the use of a simulant model to the relationship between the time flow and pressure profiles at the outlets of the simulation as a way to systematically generate appropriate outlet boundary conditions for the FLUENT simulation that are also compatible to the conditions determined downstream in the arterial network, following a 1D model approximation as developed in Johnson et al. [(2011b)]. In this way, the need for the development of a special, in-house-developed, code in order to implement those outlet boundary conditions to the 3D simulation is avoided. The simulant model is developed based on lubrication approximation. As this cannot fully account for the 3D and fully time-dependent (inertial and curvature) flow effects a correction term is also necessary. This correction term needs to be evaluated iteratively based on a comparison between the simulant and FLUENT predictions. The need of iterations brings forward the issue of convergence and efficiency for this proposed outlet boundary code conditions scheme. Although in the original work of Johnson et al. [2011a], the indications were that the iterations converge sufficiently fast to be effective, there have not been carried out to full convergence and they only have been demonstrated in a rather simplistic geometry and only with a Newtonian fluid model. We attempt to rectify all these drawbacks here while simultaneously putting forward a robust, 
Shanks transformation-based, convergence acceleration scheme. To better understand it, a brief description of the simulant model and its implementation is offered here and in the next section, respectively. The corresponding convergence acceleration through the Shanks transformation is discussed in Section 2.3.3 while all its effects in accelerating the convergence of the iterations follow, along with the results from a systematic numerical convergence study, in Section 3.

To construct the simulant model, we take first advantage of the incompressibility condition that allows for a reference pressure to be defined arbitrarily in time. Therefore, the outlet BCs can be simplified by using one of the outlet pressures as a reference and subtracting it from all other pressures. In our simulations the LAD outlet pressure is chosen as a reference ( $P_{L A D}=0$ ). Then, the outflow conditions are fully specifies by just defining the relative outlet pressure:

$$
\Delta P=P_{L C X}-P_{L A D}
$$

It is the proper specification of Eq. (6) that requires the development of the simulant model. The simulant model uses the lubrication approximation to a pressure driven Newtonian flow (Poiseuille) in each of the vessels, with only linear viscous forces to overcome. The mathematical formulation for this approximation is:

$$
\begin{gathered}
u_{j}(t, z)=u_{M, j}(t, z) \cdot\left(1-\frac{r}{R_{j}(z)}\right)^{2}, \\
\frac{d P_{j}}{d z}=\mu\left[\frac{1}{r} \frac{d}{d r}\left(r \frac{d u_{j}}{d r}\right)\right]=-\frac{8 \mu Q_{j}}{\pi\left(R_{j}(z)\right)^{4}} \Rightarrow \\
P_{j, 2}-P_{j, 1}=-\frac{8 \mu Q_{j}}{\pi} \int_{0}^{L_{j}} \frac{d z}{\left(R_{j}(z)\right)^{4}}
\end{gathered}
$$

where $U$ is the velocity, $\mathrm{P}$ the pressure, $\mu$ the Newtonian viscosity, $Q$ the flowrate, $L$ the length of the vessel, and $R$ its diameter. $P_{j, 1}$ and $P_{j, 2}$ is the pressure at the beginning (point 1) and the end (point 2) of the $\mathrm{j}$ bifurcating artery, (LCX or LAD), respectively (see Fig. 1).

For the physiological geometry of the left main coronary artery represented in Fig. 1, an analytical expression for Eq. (8) can be obtained by assuming a linear tapering of the vessels and equal pressures at the branching point of the vessel $\left(P_{L C X, 1}=P_{L A D, 1}\right)$. We also use the continuity 
equation between the total inlet flux, $Q_{\text {inl }}$ and the fluxes at each one of the two bifurcating branches, $Q_{L C X}$ and $Q_{L A D}$, respectively

$$
Q_{i n l} \equiv Q_{L A D}+Q_{L C X} .
$$

Then, evaluating Eq. (8) at each one of the LAD and LCX sections and subtracting the results leads to the following expression

$$
2 \Delta P(t)=-\alpha \Delta Q(t)+\beta Q_{i n l}(t),
$$

where $\alpha$ and $\beta$ are constants and a function of geometrical parameters and the viscosity, given by:

$$
\begin{aligned}
& \alpha=\frac{8 \mu}{3 \pi}\left[L_{L A D}\left(\frac{R_{L A D, 1}^{2}+R_{L A D, 1} R_{L A D, 2}+R_{L A D, 2}^{2}}{R_{L A D, 1}^{3} R_{L A D, 2}^{3}}\right)+L_{L C X}\left(\frac{R_{L C X, 1}^{2}+R_{L C X, 1} R_{L C X, 2}+R_{L C X, 2}^{2}}{R_{L C X, 1}^{3} R_{L C X, 2}^{3}}\right)\right], \\
& \beta=\frac{8 \mu}{3 \pi}\left[L_{L A D}\left(\frac{R_{L A D, 1}^{2}+R_{L A D, 1} R_{L A D, 2}+R_{L A D, 2}^{2}}{R_{L A D, 1}^{3} R_{L A D, 2}^{3}}\right)-L_{L C X}\left(\frac{R_{L C X, 1}^{2}+R_{L C X, 1} R_{L C X, 2}+R_{L C X, 2}^{2}}{R_{L C X, 1}^{3} R_{L C X, 2}^{3}}\right)\right]
\end{aligned}
$$

with the subscripts 1,2 referring to the inlet and outlet values, respectively, as given in Table 1 .

The final step in the development of the simulant model is the inclusion of a non-linear corrective term, $\delta(t)$. This term accounts for the observed differences between the lubrication approximation based simulant model and the $3 \mathrm{D}$ simulation results. Then, the final form of the simulant model becomes:

$$
2 \Delta P(t)=-\alpha \Delta Q(t)+\beta Q_{i n l}(t)-\alpha \cdot \delta(t) .
$$

Eq. (12) is then solved together with Eq. (5), as applied for each one of the LAD and LCX bifurcating branches, to provide an expression for the pressure differential $\Delta P(t)$ as a function of time that is then applied as boundary condition to the 3D and time-dependent simulation. As explained in detail in Section 2.3.2, below, the simulant is applied iteratively until convergence is reached between its predictions and the Fluent results. 


\subsubsection{Iterative implementation of OBCs using the simulant model}

The proper enforcement of Eq. (12) as an outlet BC in simulations of arterial flow requires an iterative procedure. For the first iteration, an initial guess for the pressure difference between the two outlets, $\Delta P$, is needed. The initial guess can be a time-dependent profile, e.g. with information obtained from a 1D network model, or even a constant, if such information is not available. Then, from the simulation output we can evaluate the corresponding $\Delta Q$ profile. Then, using Eq. (12) we evaluate the new correction $\delta(t)$. To facilitate the calculations, as the impedances are offered in the Fourier space from the use of the 1D model [Johnson et al. (2011b)], we then evaluate the Fourier transform of $\Delta Q, \Delta \hat{Q}$, as well as of the correction term, $\hat{\delta}_{k}$. From Eqs. (5),(6) and (12) one can then obtain an updated prediction of the flow rate, given by:

$$
\Delta \hat{Q}_{k}=\frac{\left(\hat{Z}_{L A D, k}-\hat{Z}_{L C X, k}+\beta\right) \hat{Q}_{i n l, k}-\alpha \hat{\delta}_{k}}{\hat{Z}_{L A D, k}+\hat{Z}_{L C X, k}+\alpha}
$$

Finally, after applying an inverse Fourier transformation to return to the time domain, Eq. (13) yields the new outlet pressure $\mathrm{BC}$, corresponding to the updated $\Delta Q$. The new $\Delta P$ is then the updated outlet $\mathrm{BC}$ that is used in the second iteration. The flow diagram of the described process is shown in Fig. 2. 


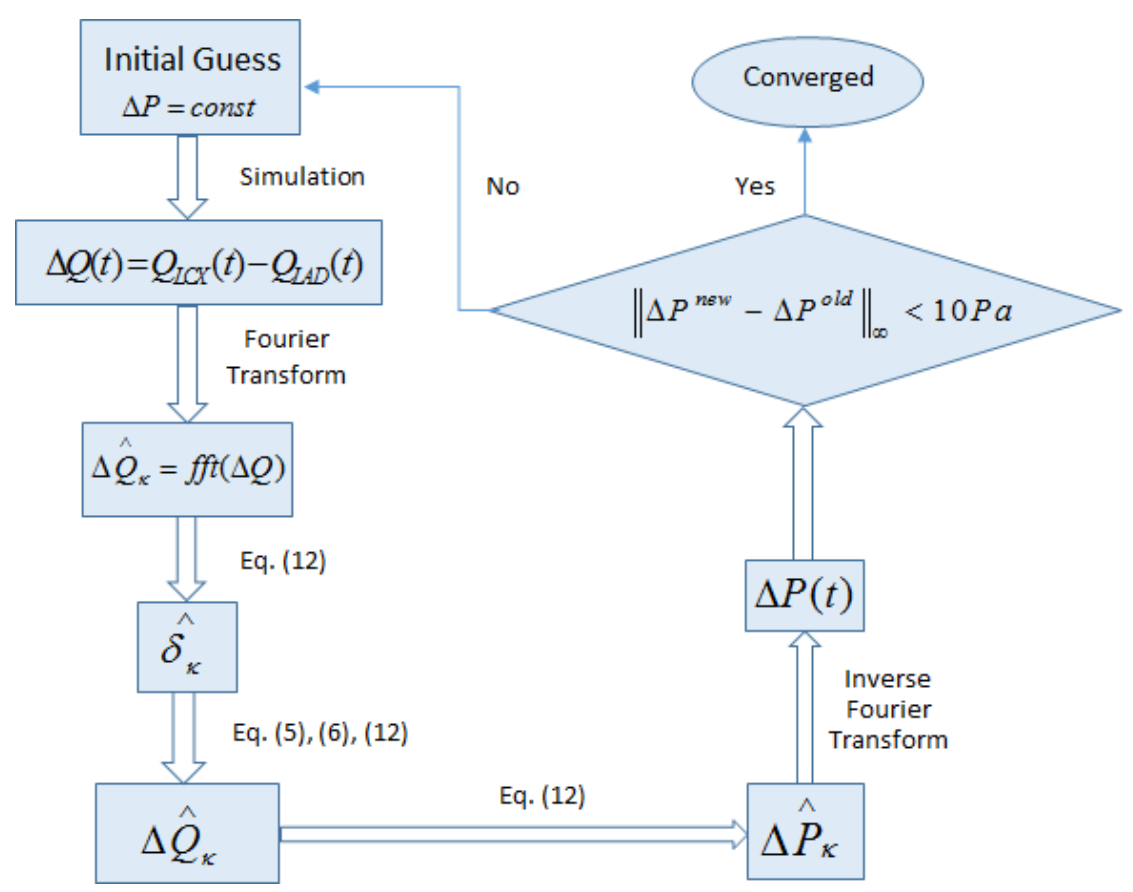

Figure 2. Logical diagram for the iterative application of the simulant model.

The proposed scheme converges in $\sim 10$ iterations, while the convergence rate is not significantly affected by the initial guess of $\Delta P$ (constant vs time dependent from 1D), which shows the robustness of the approach. However, the rather significant number of iterations required for convergence leads to the examination of numerical techniques that can potentially accelerate the convergence rate. A very efficient scheme based on Shanks transformation is described in the next section.

\subsubsection{Accelerated convergence of iterative scheme}

Numerical analysis was applied in order to accelerate the convergence rate of the proposed iterative scheme. A non-linear series acceleration method, the Shanks transformation, was used to improve the rate of convergence [Bender and Orszag (1999)]. The Shanks transformation of a sequence $A_{h}\left(A_{n}=\sum_{m=0}^{n} a_{m}\right)$ into another sequence, $S\left(A_{h}\right)$, is defined as:

$$
S\left(A_{n}\right)=\frac{A_{n+1} A_{n-1}-A_{n}^{2}}{A_{n+1}-2 A_{n}+A_{n-1}} .
$$


It can be shown [Bender and Orszag (1999)] that if the error convergence is of a power law form, i.e. $A_{n}=A_{\infty}+a q^{n}$, the transformation of Eq. (14) leads to a constant, perfectly converged, series. Of course, this rarely happens in practice, but the power law approximation can be close enough so that the transformed sequence, $S\left(A_{n}\right)$, often converges much faster than the original sequence, $A_{h}$. For the application in our system, the sequence $A_{h}$ is replaced by the $\mathrm{n}^{\text {th }}$ iteration's pressure profile, $\Delta P_{n}(t)$ and the Shanks transformation is applied to its time separately. If the initial guess of $\Delta P$ is a constant, then the minimum number of iterations required before the Shanks transformation can be applied is four. Then, the Shanks transformation yields:

$$
S\left(\Delta P_{3}(t)\right)=\frac{\Delta P_{4}(t) \Delta P_{2}(t)-\Delta P_{3}(t)}{\Delta P_{4}(t)-2 \Delta P_{3}(t) \Delta P_{2}(t)}
$$

The results of the application of the Shanks transformation in accelerating the convergence of the application of the simulant model are discussed in the first subsection of the next section.

\section{Results and Discussion}

We present a comparison between the results of the flow simulations corresponding to either the base Newtonian or the more physically relevant Casson fluid model, for the parameters presented in Section 2.2, for the first bifurcation of the left main coronary artery geometry as shown in Figure 1. The numerical results have been obtained with the FLUENT software and for the proper outlet boundary conditions so that they are compatible with the downstream $1 \mathrm{~d}$ model network predictions of Johnson et al. (2011b), following their iterative implementation through the simulant model as outlined in Section 2.3. To enhance their physical significance, we focus our comparison to two characteristic times to correspond to either the peak systole, reached at time $\left(\mathrm{t}_{1}\right)$ of $0.0625 \mathrm{sec}$, or to the late diastole instant, corresponding to a time $\left(\mathrm{t}_{2}\right)$ of $0.6875 \mathrm{sec}$ (see Fig. 3a, where also the input flow rate that we used in the simulations is indicated). We also focus on two locations corresponding to cross-sections $A_{1}$ and $A_{2}$, located along the LAD and the LCX artery, respectively, as shown in Fig. $3 b$. 
(a)

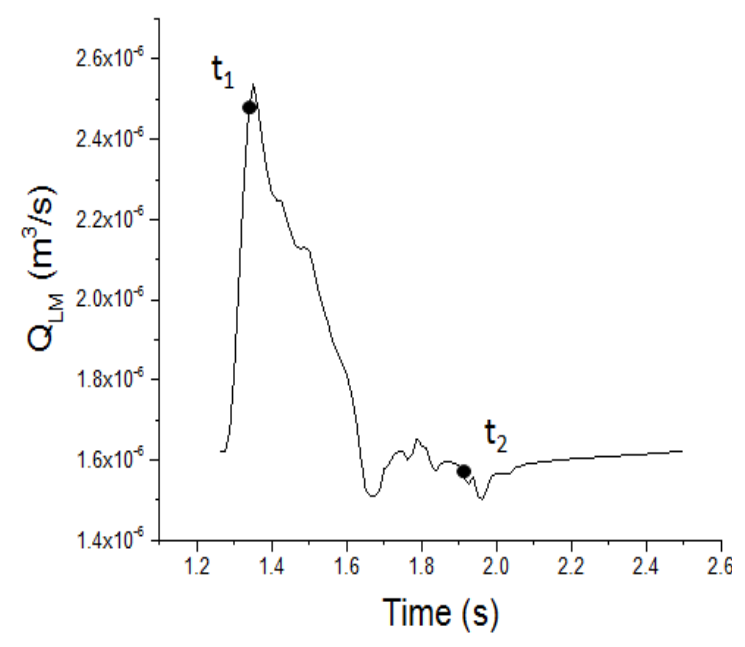

(b)

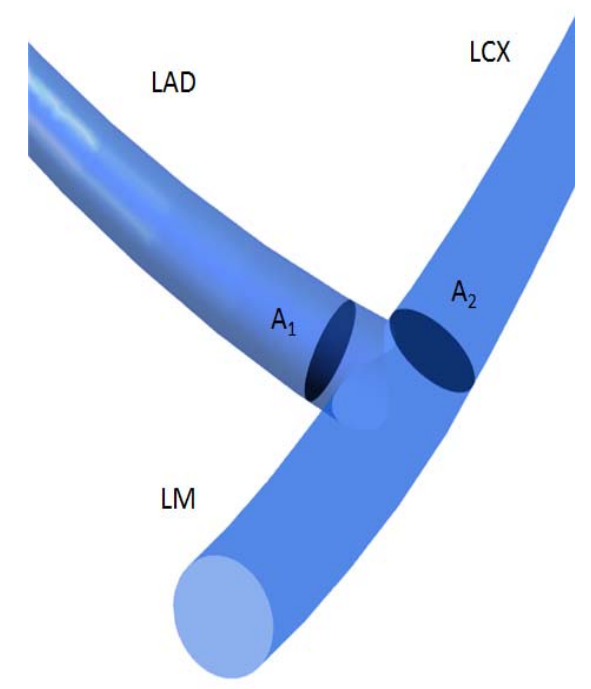

Figure 3. (a): The periodic, transient volumetric flow rate at the left main artery that is used as the inlet boundary condition [Johnson et al. (2011a)]. The peak systole and late diastole times corresponding to the second period $(1.25-2.5 \mathrm{sec}), \mathrm{t}_{1}$ and $\mathrm{t}_{2}$, respectively, are marked, (b): cross-sections $A_{1}$ and $A_{2}$, near the bifurcation site, that are used for the postprocessing of the simulation results.

An inherent component of our investigation was the evaluation and demonstration of the convergence of the numerics. A particular concern was the iterative implementation of the outlet boundary conditions, so this is examined separately first, in Section 3.1. Once this is demonstrated, we then focus on the comparison of the overall convergence of the numerical simulation based on a systematic refinement of the numerical resolution in both space and time. The results of this investigation are shown and discussed in Section 3.2. The presentation and analysis of the detailed comparison of the results obtained for a Newtonian and a Casson fluid then follow in Section 3.3.

\subsection{Convergence of iterative scheme for outlet boundary conditions}

Based on the numerical analysis described in section 2.3.3, the Shanks transformation was applied (usually in the $5^{\text {th }}$ or the $6^{\text {th }}$ simulation) to get an accelerated convergence of the application of the outlet boundary conditions, imposed through the iterative scheme discussed in Section 3. To examine the implications of that application, we follow in Fig. 4 the development 
of the differential pressure $\Delta P$ and differential flow rate $\Delta Q$ between the LCX and LAD outlets as they were recorded in our flow simulations using FLUENT and the base mesh and time-step values reported in Table 2 in section 3.2 using the Newtonian viscosity model---the convergence results were very similar under all other mesh and model conditions.
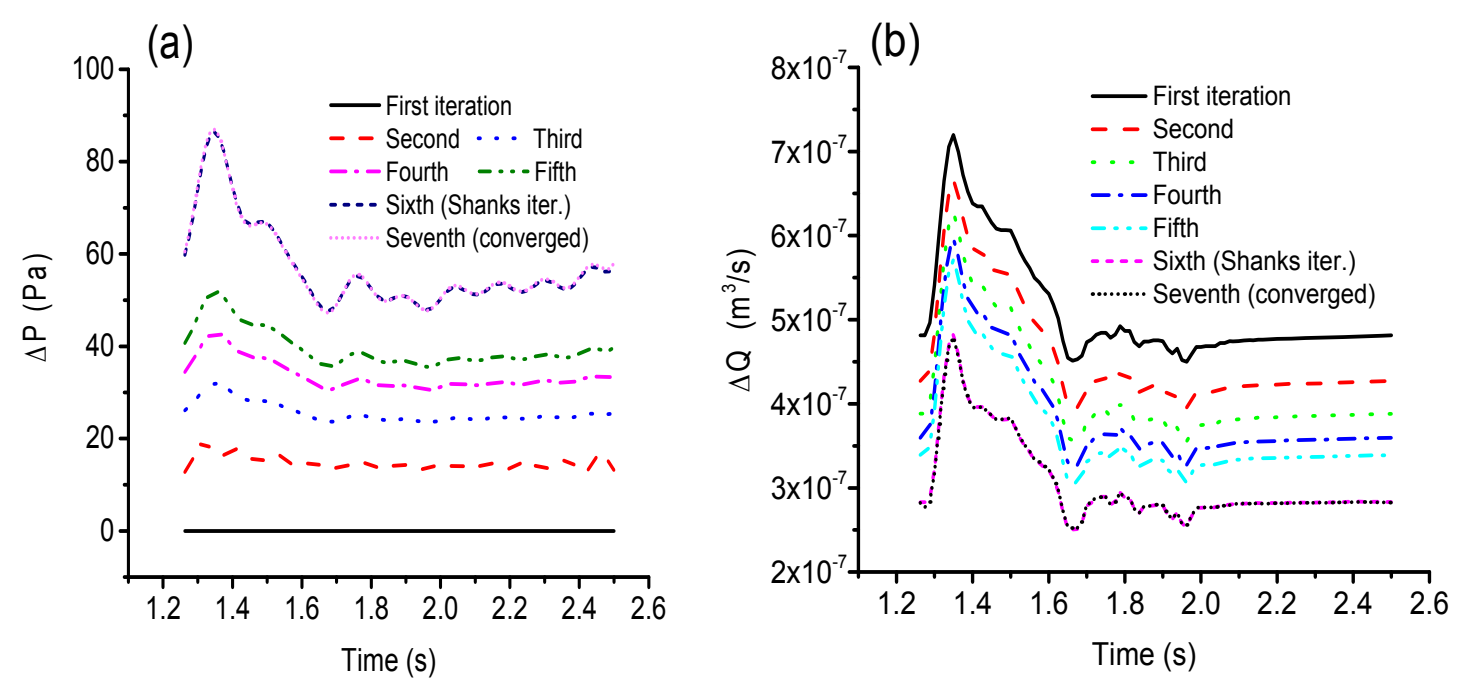

Figure 4. Outlet differential pressure (a) and differential flowrate (b) profiles of Newtonian-based simulations as obtained from the iterative procedure outlined in Section 2.3 using the base mesh and time-step values as shown in Table 2.

As clearly seen for the results presented in Fig. 4, the Shanks transformation accelerates the convergence of both the pressure and flow profiles with a convergence established (to within differences imperceptible upon a visual examination of the results) right after its first application, implemented here at the sixth iteration. Depending on the simulation specifications (mesh density, integration time-step size, iterations per time step), the iterative scheme alone requires between 10-18 iterations for convergence (data not shown). The Shanks transformation reduces therefore the required number of iterations by $30 \%-60 \%$. Based on these results, we conclude that the Shanks transformation can consistently accelerate the convergence rate of the proposed iterative scheme, and therefore can significantly reduce the computational demands of the analysis. It has therefore been used in all the results reported here.

Looking at the physical nature of the reported results in Fig.4, it is important to note that upon the imposition of the consistency with the downstream arterial network flow behavior as the criterion for outlet conditions, those develop from the more commonly used zero differential 
pressure assumption into a quite significant and a-priori not easy to predict profile. Not only is the differential outlet pressure between the LCX and LAD branches substantially different than zero (and of the same magnitude as the overall pressure difference observed along anyone of the three arterial vessels simulated here) but also it shows a significant time variability closely following the overall applied pressure pulse. This is also accompanied by a significant differential flux between the two branching levels. This feature, characteristic of the branching asymmetry investigated here, is a significant component of the flow simulation and cannot be neglected. Moreover, it is only anticipated to intensify when additional effects are to be studied, such as those of a stenosis, that significantly alter the flux balances. The outlet boundary conditions dependence to the outlet network becomes then even more crucial.

\subsection{Numerical convergence}

It is common practice in CFD investigations is to perform a convergence study i.e. sensitivity analysis to the key numerical parameters involved, in order to show that the obtained results are not significantly affected by numerical error. In the vast majority of the medical related CFD studies in literature, this analysis is restricted to the sensitivity of the simulation outcome to spatial meshes of different densities. Moreover, the sensitivity is often tested only under steady state conditions. Such approaches neglect the impact of the pulsatile nature of blood flow on the numerical stability of the simulations, while the sensitivity to parameters other than the mesh density, such as the integration time step, is rarely examined. Performing a more holistic sensitivity analysis is crucial not only for the obvious reason, that of reliability of the yielded results, but also because, despite the decrease of hardware costs, the simulations of flow in vasculature remain computationally expensive, therefore adopting more refined parameters than necessary can lead to a significant increase in time and/or computational needs.

We have adapted hare a more comprehensive convergence analysis by systematically examining the impact of the refinement both in the spatial mesh density, as well as the integration time-step and the number of iterations allowed per time-step. We are considering three progressively refined cases of parameter sets: a coarse, a base, and a dense one. The analysis is not performed only on the Newtonian-based simulations but also on simulations based on the Generalized Newtonian (Casson) model, in order to ensure that the additional nonlinearity of the that model does not influence the numerical stability. The parameters used in each case are presented in Table 2. Also note that to ensure that any transient effects have died away we always compare results obtained at the second period of the applied periodic inlet flow. It is also of importance to note that we only compare converged results with respect to the implementation of the outlet boundary conditions, as shown in Section 3.1 and presented in Fig. 4. The converged pressure and flux profiles for all six cases (three physiological-Newtonian, three physiological-Casson) are shown in Fig. 5. 
Table 2. Parameters used for the numerical convergence analysis.

\begin{tabular}{lccc}
\hline \hline & Coarse & $\begin{array}{c}\text { Parameter sets } \\
\text { Base }\end{array}$ & Dense \\
\hline Number of cells & 43,572 & 123,112 & 244,918 \\
Time-step & 0.025 & 0.0125 & 0.00625 \\
Number of steps & 100 & 200 & 400 \\
Iterations per time-step & 40 & 80 & 160 \\
Period & 2 & 2 & 2 \\
\hline \hline
\end{tabular}

(a)

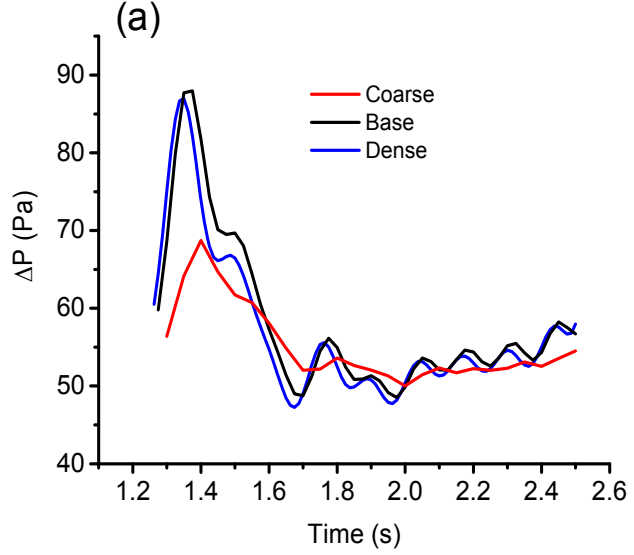

(c)

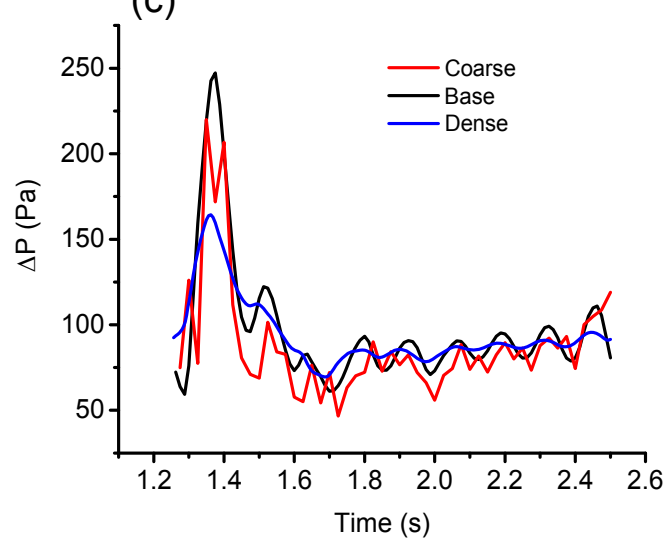

(b)
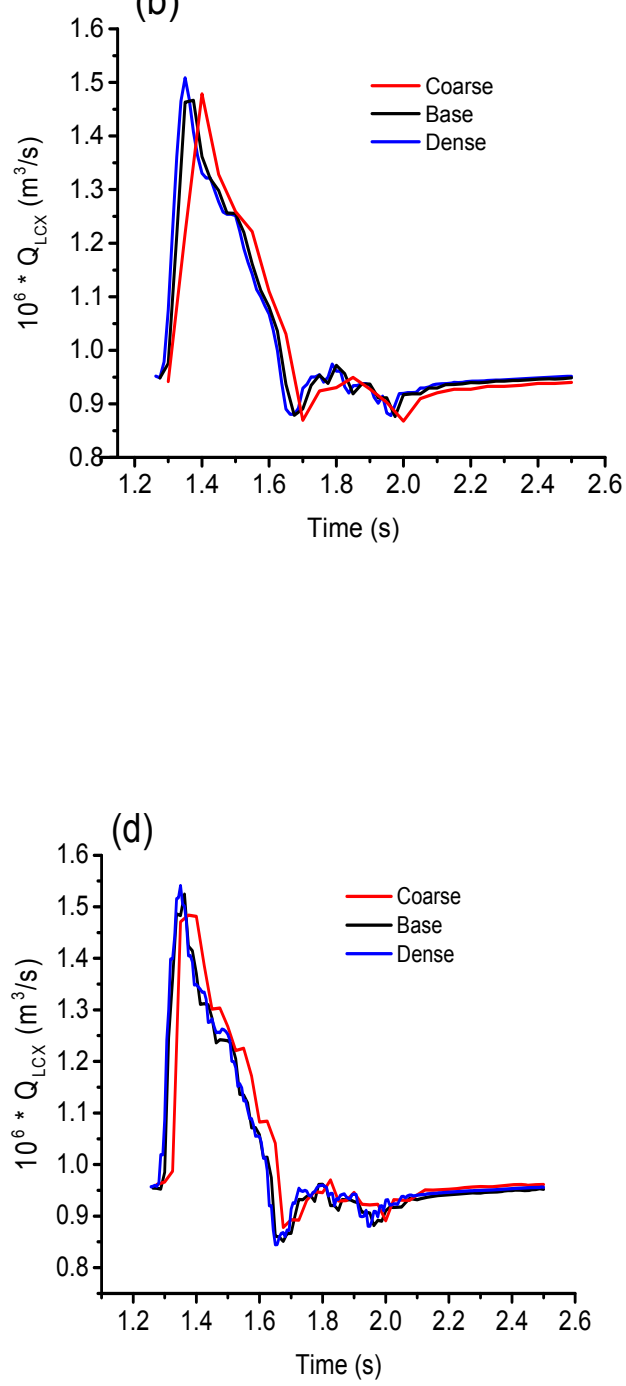
Figure 5. Comparison of the converged differential outlet pressure profiles $(\mathrm{a}, \mathrm{c})$ and LCX outlet flow rates $(b, d)$ for a Newtonian fluid $(a, b)$, and a Casson fluid $(c, d)$.

It is of importance to note here some significant differences observed in those convergence studies, especially between the Newtonian (Fig.5 a,b) and the Casson (Fig. 5 c, d) cases. First, the Newtonian cases appear to converge faster, with the difference between the base and dense cases being minimal, both with respect to the average as well as the overall time-profiles. Second, while there is a similar good convergence in the flowrate results of the Casson cases, the pressure time profiles showed significant variations and especially oscillations around the systolic peak, first the pressure exhibiting an unusual double peak with the coarser mesh, then an overshoot with the base case, before settling in a more reasonable profile with the dense mesh conditions. Clearly, the additional nonlinearities introduced by the Casson model require additional refinements to both the spatial and time discretizations to provide converged results. Still, looking at the average value of the differential pressure obtained in the three different cases (obtained as $87.7 \mathrm{~Pa}, 97.01 \mathrm{~Pa}$, and $94.43 \mathrm{~Pa}$ for the coarse, base and dense cases, respectively) there is a clear convergence. The faster convergence on the flowrate can be explained from the fact that it is the overall inlet flowrate that it is imposed (the same) in all cases, whereas the pressure simply adjusts to the solution and the pressure outlet conditions.

A final remark is warranted on the physical meaning of the reported results. Namely, in comparing the results between the Newtonian and the Casson model, it is very striking that there are considerable differences observed in the differential pressure profile: Not just the mean but also (and especially) the peak differential pressure values show significant differences, with the non-Newtonian results being as much as $50 \%$ higher. This is a first, but clear, indication of the significance that Non-Newtonian effects may have in blood flow simulations. We will see more

of those and discuss them in higher details in the detail comparison presented in the following section.

\subsection{Comparison between the Newtonian and Casson Fluid Results}

In the reported results here we rely on converged solutions obtained using the dense case numerical parameters, as specified in Table 2. The peak systole results are obtained for comparison purposes, as, from a physiological viewpoint, we are mostly interested in the late diastole findings. The non-Newtonian rheology is expected to be more prominent at low shear rates, which is when the stress and velocity profiles are at a minimum. The same reasoning holds for the selection of the particular locations of the cross-sections $A_{1}$ and $A_{2}$. Near the bifurcation site we expect the formation of recirculation zones (due to flow separation), and therefore the appearance of zones of low shear rates. This observation is also consistent the correlation of low shear stress regions with the anticipated locations of formation of atherosclerotic plaques that as explained in the introduction have a tendency to develop that low 
shear stress. This explains further the interest to describing accurately the non-Newtonian blood rheology.

The comparison between Newtonian and Casson simulations shows important differences between the two cases. To compare the two models we have plotted in Fig. 6 the wall shear stress (WSS) distribution along the circumference of the cross-sections $A_{1}$ and $A_{2}$, as predicted in each case (Newtonian vs Casson).
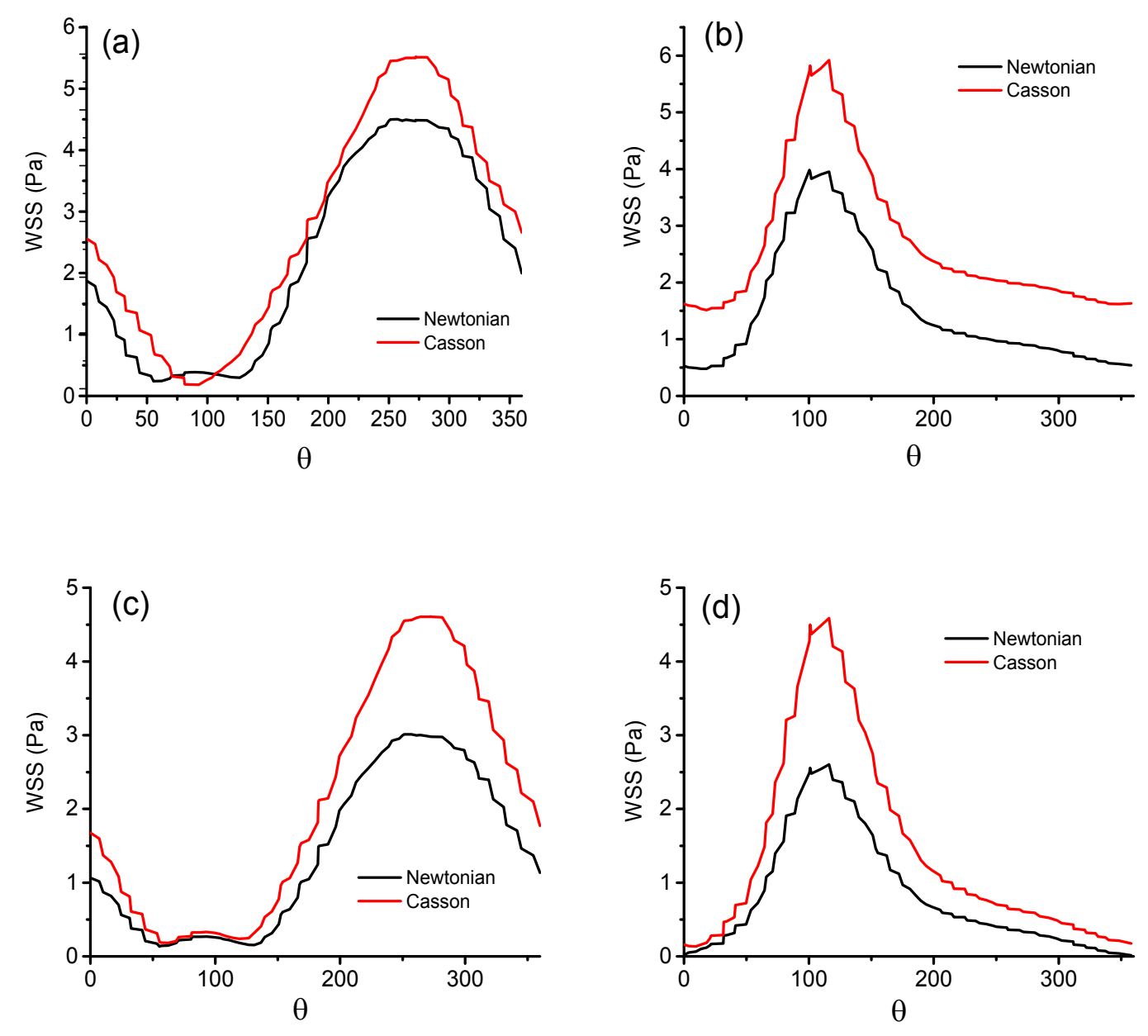

Figure 6. WSS distribution along the circumference of the cross-sections $A_{1}(a, c)$ and $A_{2}(b, d)$, evaluated at $t_{1}(a, b)$ and $t_{2}(c, d)$. Note that the jagged nature of the results is a consequence of the discrete (finite volume) numerical approximation used and not a defect of poor resolution graphics.

Fig. 6 clearly demonstrates that the Newtonian predictions of WSS are consistently lower to those of the Casson model. This conclusion is in agreement with the predictions of Karimi et al. (2014) for the WSS distribution in the human aorta. In addition, we notice that for the 
(a)
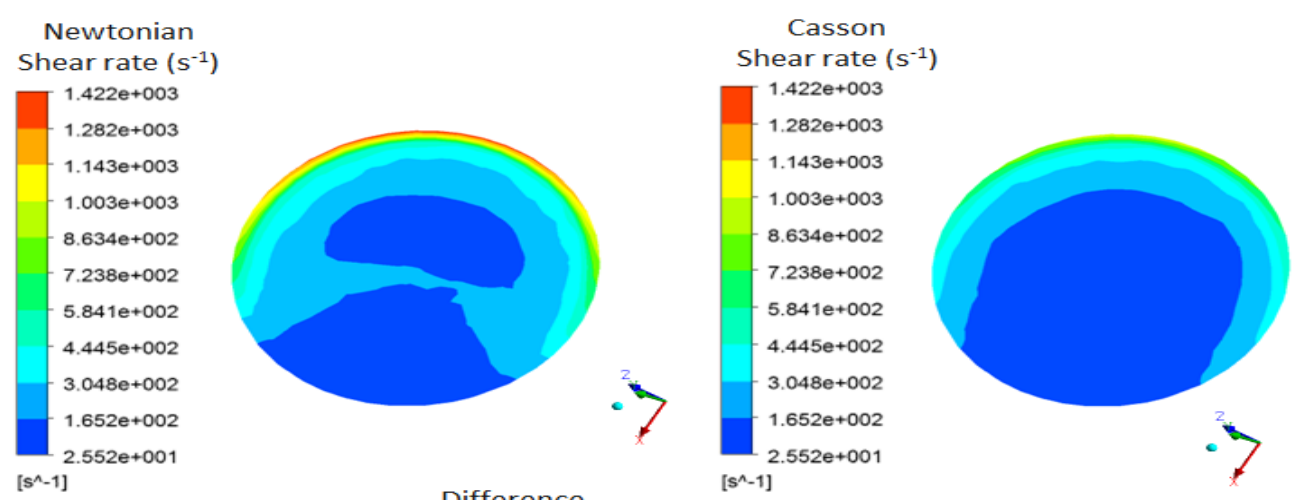

Shear rate $\left(\mathrm{s}^{-1}\right)$
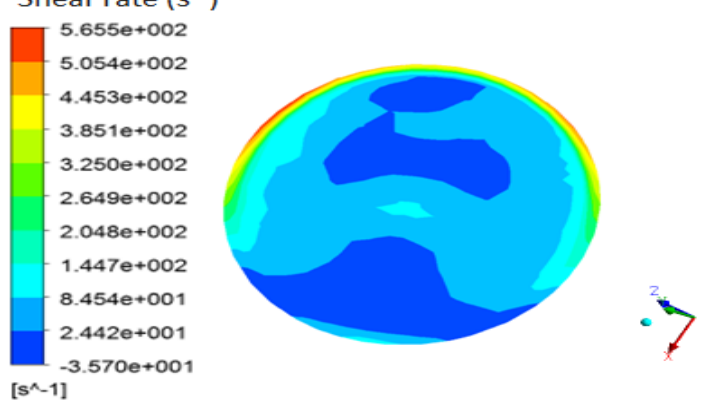

(b)
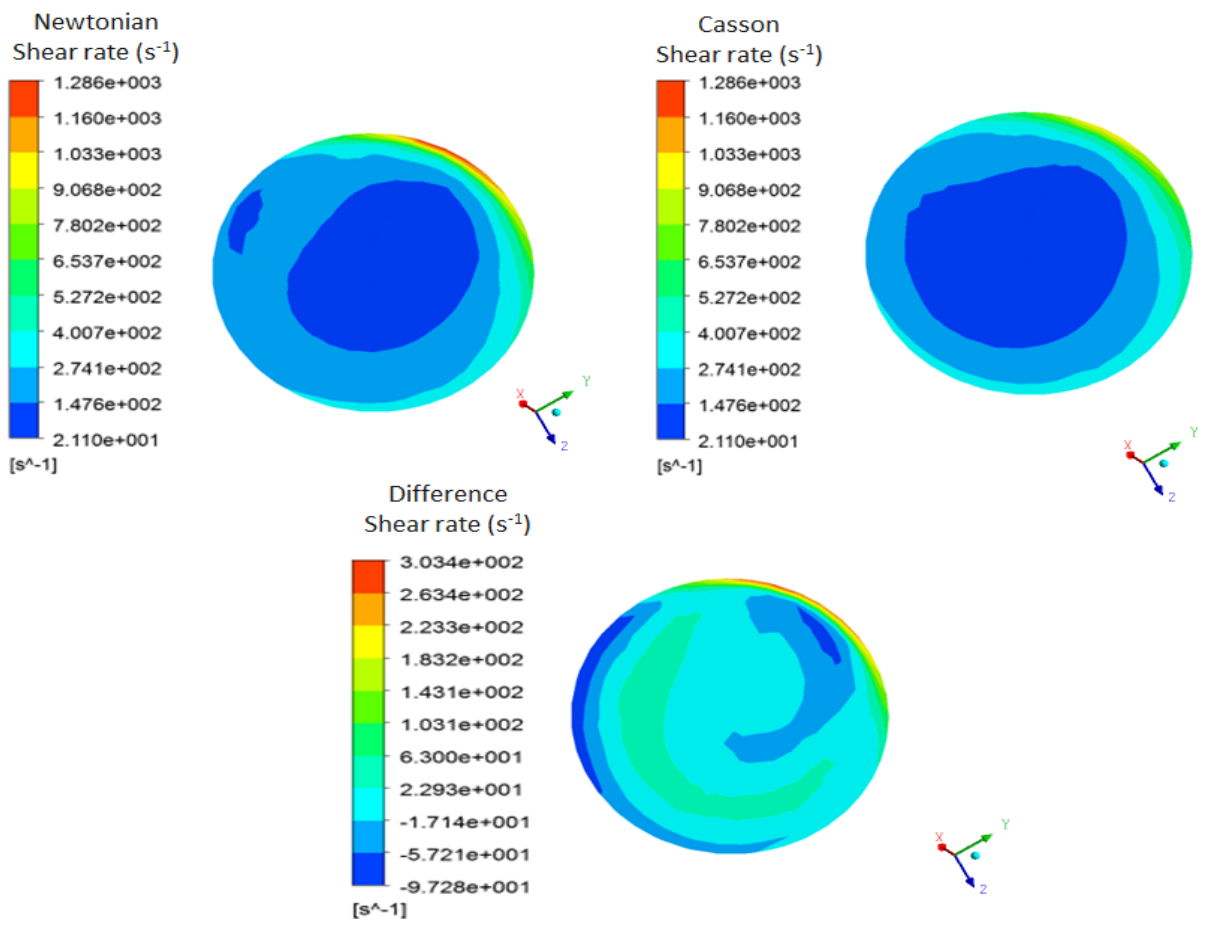

Figure 7. Shear rate contours of planes $A_{1}$ (a) and $A_{2}(b)$, evaluated at $t_{2}$ (the diastolic time), with the Newtonian (left) and the parametric Casson (right) models, and their difference (bottom). 
circumference of both cross-sections the maximum deviation between the WSS predictions of the two models occurs, in both cases, at the late-diastole time, t2 (Fig. 6 (c-d)). This result is attributed to the fact that the non-Newtonian rheology is primarily manifested at lower shear rates. The maximum differences also occur when the wall shear stresses assume their highest (peak) values, where the Casson predictions can be almost twice that of the Newtonian fluid. In contrast, near the positions when the shear stresses assume their lowest values the two models almost coincide- especially in the LAD cross section---see Figs. 6(a) and 6(c).

Significant deviations between the Newtonian and the Casson model predictions were also apparent when comparing the predicted shear rate values along the two cross-sections. The contours of shear rate for the two planes $A_{1}$ and $A_{2}$ are shown in Fig. 7. The maximum difference in shear rate estimations of the two models at cross-sections $A_{1}$ and $A_{2}$ is $40 \%$ and $24 \%$, respectively. Thus, from the illustrative results of Figs. 6-7 it can be concluded that the rheology of blood impacts significantly the simulation outcome even in this case where the encountered shear rate values, according to the contour values in Fig. 7, are significantly higher than the regime of one reciprocal second where most of the differences between the two models are naturally located. This gives special value to using appropriate rheology models and model parameter values in blood flow simulations.

\section{Conclusions}

We have presented a careful evaluation of non-Newtonian blood rheology effects in a realistic simulation of arterial flow in the first bifurcation of the left main coronary artery. We did that evaluation by comparing the converged solutions obtained for a non-Newtonian viscoplastic model, the Casson model, and a Newtonian one. We evaluated the Casson model yield stress and viscosity parameter exploiting recently developed correlations with blood physiological parameters, for which we used normal physiological values, whereas with the Newtonian model we used a standard blood viscosity value---interestingly enough its value came very close to the Casson model viscosity value isolating the differences between the two models in the presence of a non-zero yield stress in the Casson case. Special care has been paid to make sure that in the vessels geometry both the naturally occurring vessels tapering as well as vessels curvature are represented, whereas appropriate outlet boundary conditions ascertain the consistency of the outlet flow and pressure values to those determined based on the downstream vessels of the arterial network. Special care has also been paid to make sure that for the numerical parameters used in the comparisons the solutions have appropriately converged.

The most significant result of the comparisons is that there are important quantitative differences observed between the solutions obtained with the two different rheological models. 
Those differences can be as much as $50 \%$ a value surprisingly large given the preponderance of high shear rates in the simulated arterial flow, while the non-Newtonian effects are primarily manifested at the low shear rate regime. The differences therefore need to be attributed to the coupling that exists between low and high shear rate areas in the flow. This coupling we consider it that it is especially affected by the shear thinning characteristics of the Casson model that within the complex and time-dependent flow field are completely overshadowing any yield stress effects. They are consistent with similar findings reported in the literature and they clearly demonstrate the importance of utilizing the correct rheology and appropriate rheological parameters in blood flow simulations if quantitative predictions are to be made. They also motivate further study of blood flow rheology that can possibly also incorporate appropriate for describing the blood thixotropy time-history effects as well as models that are valid for general three-dimensional flows, as most flows (including the one studied here) are also time-dependent and fully three-dimensional. The present study therefore can be considered as providing an upper bound of the anticipated non-Newtonian effects to this coronary arterial flow, as those thixotropic effects are, in general, anticipated to lower the viscoplastic characteristics predicted by the non-Newtonian Casson model (although given the coupling between the different regions demonstrated in this work and the fact that following transient from a low to a high flow deformation region one may anticipate a reverse thixotropic effect one may not necessarily know for sure the effects of thixotropy before a full consistent calculation is carried out). It is also to be noted that the differences may even be greater in flows where lower shear rates are encountered.

In parallel with this investigation of the non-Newtonian effects of blood rheology to blood flow simulations we also provided a further validation of an efficient implementation of proper outlet BCs in simulations of blood flow arterial flows that we have developed in the past but so far not extensively tested. This methodology, general enough to allow the use of commercial software with only standard outlet conditions as option (such as FLUENT used here), applies impedance relations between the flowrate and pressure at its outlet, as they are developed from a 1d approximate arterial network flow model, within a simulant model of the actual outlet pressure for a give flowrate, in order to generate pressure outlet conditions that can be applied to the 3D CFD simulations. We have followed the methodology, first proposed by Johnson et al. (2011a), albeit we have: (a) applied the analysis to a more realistic geometrical model, (b) used the Shanks transformation to accelerate the convergence of the iteratively applied simulant model, and (c) performed a holistic numerical sensitivity convergence analysis to ensure that the displayed simulation results are not significantly affected by numerical error.

\section{Acknowledgments}

This material is based upon work supported by the National Science Foundation under Grant No. CBET 1033296. Any opinions, findings, and conclusions or recommendations expressed in this 
material are those of the author(s) and do not necessarily reflect the views of the National Science Foundation. The authors will also like to acknowledge the help of Rohith Venkataraman in developing the methodology that is followed for the convergence studies performed in this work and many helpful discussions with Dr. David Johnson.

\section{References}

Apostolidis, A. J., and A. N. Beris, "Modeling of the blood rheology in steady-state shear flows," J. Rheol. 58, 607-633 (2014).

Apostolidis, A. J., M. J. Armstrong, and A. N. Beris, "Modeling of human blood rheology in transient shear flows," J. Rheol. 59, 275-298 (2015).

Bender, C. M., and S. A. Orszag, Advanced Mathematical Methods for Scientists and Engineers: Asymptotic Methods and Perturbation Theory, Springer: New York (1999).

Bernabeu, M. O., R. W. Nash, D. Groen, H. B. Carver, J. Hetherington, T. Krüger, and P. V. Coveney, "Impact of blood rheology on wall shear stress in a model of the middle cerebral artery," Interface Focus 3, 20120094 (2013).

Bureau, M., J. C. Healy, D. Bourgoin, and M. Joly, "Rheological properties of blood at low shear rate," Biorheology 17, 191-203 (1980).

Byoung-Kwon, L. “Computational Fluid Dynamics in Cardiovascular Disease,” Korean Circulation Journal 41, 423-430 (2011).

Campo-Deaño, L., M. S. N. Oliveira, and F. T. Pinho, “A review of computational hemodynamics in middle cerebral aneurysms and rheological models for blood flow," Appl. Mech. Rev. 67 (3), 1-16 (2015).

Cecchi, E., C. Giglioli, S. Valente, C. Lazzeri, G. F. Gensini, R. Abbate, and L. Mannini, "Role of hemodynamic shear stress in cardiovascular disease," Atherosclerosis 214, 249-256 (2011).

Chaichana, T., S. Zhonghua, and J. Jewkes, "Computational fluid dynamics analysis of the effect of plaques in the left coronary artery," Comput. Math. Methods Med. 2012:504367 (2012).

Dodge, J.T. Jr., B. G. Brown, E. L. Bolson, H. T. Dodge, "Lumen diameter of Normal Human Coronary Arteries. Influence of age, sex, anatomic variation, and left ventricular hypertrophy or dilation," Circulation 86, 232-246 (1992).

Dong, J., Z. Sun, K. Inthavong, and J. Tu, "Fluid-structure interaction analysis of the left coronary artery with variable angulation," Comput. Methods Biomech. Biomed. Engin. 18(14), 1500-1508 (2015). 
Esmaily-Moghadam, M., I. E. Vignon-Clementel, R. Figliola, and A. L. Marsden, “A modular numerical method for implicit 0D/3D coupling in cardiovascular finite element simulations," Journal of Computational Physics 244, 63-79 (2013).

Formaggia, L., A. Quarteroni, and A. Veneziani (eds). "Cardiovascular Mechanics; Volume 1. Modeling and Simulation of the Circulatory System," Springer: Italia, Milano (2009).

Formaggia, L., J. F. Gerbeau, F. Nobile, and A. Quarteroni, "On the coupling of 3D and 1D Navier-Stokes equations for flow problems in compliant vessels," Computational Methods on Applied Mechanics and Engineering 191, 561-582 (2001).

Johnson, D. A., U. P. Naik, and A. N. Beris, "Efficient implementation of the proper outlet flow conditions in blood flow simulations through asymmetric arterial bifurcations," International Journal for Numerical Methods in Fluids 66, 1383-1408 (2011a).

Johnson, D. A., J. R. Spaeth, W. C. Rose, U. P. Naik, and A. N. Beris, “An impedance model for blood flow in the human arterial system. Part I: Model development and MATLAB implementation," Computers and Chemical Engineering 35, 1304-1316 (2011b).

Johnston, B. M., P. R. Johnston, S. Corney, and D. Kilpatrick, "Non-Newtonian blood flow in human right coronary arteries: Transient simulations," Journal of Biomechanics 39, 11161128 (2006).

Karimi, S., M. Dabagh, P. Vasana, M. Dadvar, B. Dabir, and P. Jalali, "Effect of rheological models on the hemodynamics within human aorta: CFD study on CT image-based geometry," Journal of Non-Newtonian Fluid Mechanics 207, 42-52 (2014).

Lassaline, J. V., and B. C. Moon, “A computational fluid dynamics simulation study of coronary blood flow affected by graft placement,” Inter. Cardiovasc. Thorac. Surg. 1-5 (2014).

Liu, X., Y. Fan, X. Deng, and F. Zhan, "Effect of non-Newtonian and pulsatile blood flow on mass transport in the human aorta,” J. Biomech. 44, 1123-1131 (2011).

Morbiducci, U., R. Ponzini, D. Gallo, C. Bignardi, and G. Rizzo, "Inflow boundary conditions for image-based computational hemodynamics: impact of idealized versus measured velocity profiles in the human aorta," J. Biomech. 46, 102-109 (2013).

Olufsen, M. S., "Modeling Flow and Pressure in the Systemic Arteries", Applied Mathematical Models in Human Physiology, J.T. Ottesen, M.S. Olufsen, and J.K. Larsen (eds.), SIAM: Philadelphia (2004).

Soulis, J. V., G. D. Giannoglou, Y. S. Chatzizisis, K. V. Seralidou, G. E. Parcharidis, and G. E. Louridas, "Non-Newtonian models for molecular viscosity and wall shear stress in a 3D reconstructed human left coronary artery," Med Eng Phys 30, 9-19 (2008). 
Taylor, C.A., T.J.R. Hughes, and C.K. Zarins, "Finite element modeling of three-dimensional pulsatile flow in the abdominal aorta: Relevance to atherosclerosis," Annals of Biomedical Engineering 26, 975-987 (1998).

Wentzel, J. J., Y. S. Chatzizisis, F. J. Gijsen FJ, G. D. Giannoglou, C. L. Feldman, and P. H. Stone, "Endothelial shear stress in the evolution of coronary atherosclerotic plaque and vascular remodelling: current understanding and remaining questions," Cardiovasc. Res. 96, 234-243 (2012).

West, G. B., "The Origin of Scaling Laws in Biology," Physica A. 263, 104-113 (1999).

Womersley, J. R., "Oscillatory Motion of a Viscous Liquid in a Thin-Walled Elastic Tube-I: The Linear Approximation for Long Waves," Phil. Mag. 46, 199-221 (1955), 